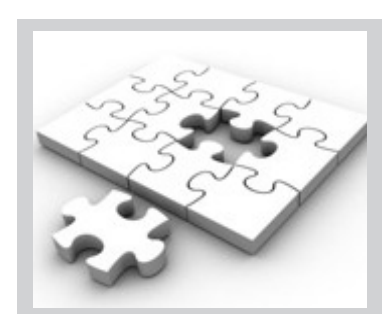

In an effort to facilitate the selection of appropriate peer reviewers for the African Journal of Primary Health Care \& Family Medicine, we ask that you take a moment to update your electronic portfolio on https://phcfm.org for our files, allowing us better access to your areas of interest and expertise, in order to match reviewers with submitted manuscripts.

If you would like to become a reviewer, please visit the journal website and register as a reviewer.

To access your details on the website, you will need to follow these steps:

1. Log into the online journal at https:// phcfm.org

2. In your 'user home' [https://phcfm.org/index. $\mathrm{php} / \mathrm{phcfm} / \mathrm{user}$ ] select 'edit my profile' under the heading 'my account' and insert all relevant details, bio statement and reviewing interest(s).

3. It is good practice as a reviewer to update your personal details regularly to ensure contact with you throughout your professional term as reviewer to the African Journal of Primary Health Care \& Family Medicine.

Please do not hesitate to contact us if you require assistance in performing this task.

Publisher: publishing@aosis.co.za Tel: +27219752602

Tel: 0861000381

\title{
Acknowledgement to reviewers
}

The editorial team of the African Journal of Primary Health Care \& Family Medicine recognises the value and importance of the peer reviewer in the overall publication process - not only in shaping the individual manuscript, but also in shaping the credibility and reputation of our journal.

We are committed to the timely publication of all original, innovative contributions submitted for publication. As such, the identification and selection of reviewers who have expertise and interest in the topics appropriate to each manuscript are essential elements in ensuring a timely, productive peer review process.

We would like to take this opportunity to thank all reviewers who participated in shaping this volume of the African Journal of Primary Health Care \& Family Medicine. We appreciate the time taken to perform your review(s) successfully.

Abdul K.M. Hamdulay

Abraham N.I. Gyuse

Adrian Wilson

Akye Essuman

Alberto Piubello

Alero A. Roberts

Amit Chandra

Andrew Janssen

Andrew J. Ross

Andrew L. Gray

Angela A. Morris-Paxton

Angeni Bheekie

Annie Botes

Babatunde Ogunnowo

Balandeli S.I. Sonti

Benjamin Mills

Bernhard M. Gaede

Beverley R. Schweitzer

Billy M. Tsima

Bruce Dahlman

Busisiwe R. Bhengu

Charlene Downing

Charles Branas

Charles Todd

Charlyn Goliath

Claire van Deventer

Colette Gunst

Colleen Aldous

Corinna M. Walsh

Danine Kitshoff

Darcelle Schouw

David Edwards

David A. Cameron

Denon Tshienda

Dereje Habte

Derek A. Hellenberg

Dinky Levitt

Doreen K. Kaura

Edu Burgueño

Elizabeth Mason

Elize Archer

Elma de Vries

Emmanuel W. Wamalwa

Engela (Adri) A.M. Prinsloo

Eric Decloedt

Esther O. Oluwole

Fanie Serfontein

Farai Madzimbamuto

Febisola I. Ajudua

Felicity Goodyear-Smith
Fiona Makoni

Francois Coetzee

Frank A. Imarhiagbe

Gerald Shambira

Gert Marincowitz

Glad Mohesh

Gladwin Muchena

Graham Bresick

Gulnaz M. Merali

Hanneke Brits

Hannes W.J. Steinberg

Hassan Mahomed

Hassen Mamo

Heleen du Toit

Helmuth Reuter

Henry Lawson

Hilary W.J. Rhode

Hoffie Conradie

Ian Couper

Ibrahim S. Bello

Inam Chitsike

Ineke Buskens

Inis J. Bardella

Innocent K. Besigye

Jack Barker

Jacob S. Shabani

Jacqueline M. van Wyk

Jeremy Wright

Jimmy Chandia

Jody P. Mbuilu

Joleen Cairncross

Joseph Iruedo

Judy McKenzie

Katherine Murphy

Kathryn Spangenberg

Kenneth Yakubu

Kerrin Begg

Khabiso J. Ramphoma

Klaus B. von Pressentin

Langalibalele H. Mabuza

Lawson B. Eksteen

Liezl Koen

Lillian N. Mukisa

Lisa Langhaug

Louis S. Jenkins

Lushiku Nkombua

Mohammed Shoyeb

Modupe M.A. Ladipo

Manie de Klerk

Margaret Williams 
Marietjie van Rooyen

Marietjie R. de Villiers

Mark F. Cotton

Masemiano P. Chege

Maureen N. Sibiya

Mergan Naidoo

Michelle Visser

Mobolanle Balogun

Mojisola O. Owolabi

Molly Whalen-Browne

Mpundu Makasa

Munyaradzi Madhomibiro

Muriel S. Fallala

Muyibi S. Abu

Neil A. Cameron

Nelisiwe Khuzwayo

Nomathemba P. Taukobong

Nyaradzai P. Magande

Oathokwa Nkomazana

Olayinka O. Ayankogbe

Olufemi B. Omole

Olukayode A. Adeleke

Owen O. Eales

Patience Dhliwayo

Paul A. Kapp

Paul B.M. Bossyns

Per Kallestrup

Peter Delobelle

Peter C. Clarke-Farr

Prosper M. Lutala

Pugie T. Chimberengwa

Ramprakash Kaswa

Raymond Downing

Riette Burger

Rose Kambarami

Rudo Nyamakura

Ruth Cornick

Sebeka Malope
Selma Smith

Shabir Moosa

Shepherd Shamu

Silvia Carlos

Sinegugu E. Duma

Solome Bakeera

Solomon H. Mukungunugwa

Somasundram Pillay

Sophie Mogotlane

Stan Houston

Stanely Tapesana

Stephan Rabie

Stephen P. Merry

Steve Reid

Steve Swartz

Sumaya Laher

Sunanda C. Ray

Suren Sirkar

Surona Visagie

Susan CarnesChichlowska

Susan A. Nzenze

Talitha Crowley

Tasleem Ras

Tebogo Ramahlo

Tessa S. Marcus

Thandaza C. Nkabinde

Thanyani G. Lumadi

Therese Fish

Tuviah Zabow

Vanessa Lomas-Marais

Vincent Setlhare

Wayne M. Simmonds

Werner Viljoen

Whadi-Ah Parker

William B. Ventres

Xikombiso G. Mbhenyane

Zelra Malan

Zimbini Ogle 